\title{
Optical diffraction from fractals with a structural transition
}

\author{
Felipe Pérez-Rodríguez \\ Instituto de Física, Universidad Autónoma de Puebla, \\ Apdo.Post. J-48, Puebla 72570, Pue., México
}

Wei Wang

Physics Department, Nanjing University, People's Republic of China

Enrique Canessa

ICTP-International Centre for Theoretical Physics, Trieste, Italy 


\begin{abstract}
A macroscopic characterization of fractals showing up a structural transition from dense to multibranched growth is made using optical diffraction theory. Such fractals are generated via the numerical solution of the 2D Poisson and Biharmonic equations and are compared to more 'regular' irreversible clusters such as diffusion limited and Laplacian aggregates. The optical diffraction method enables to identify a decrease of the fractal dimension above the structural point.
\end{abstract}

PACS numbers: 42.20, 05.40.+j, 68.70.+w 
Fractal surfaces observed in nature can become very complex due to structural transitions that are generated during their growing processes. Examples of such phenomena can be found on bacterial colonies [1] and electrochemical deposition experiments [2]. The observed fractal patterns exhibit a similar class of complex structure despite the fact that the mechanisms involved are clearly different. It has been found that, at a certain threshold distance, these systems display an intriguing structural transition from dense to multibranched growth behaviour of which not much is yet understood.

In the current literature, two different approaches attempting to explain and reproduce a structural transition during irreversible growth have been proposed. In the first one [3, 4, 5] the transition has been derived by solving the Poisson equation on a squared lattice -that becomes dependent on the potentials at two boundaries, the distance between them, and a screening length. Hence, it has been argued that screening, due to free charges, strongly diversifies the patterns that grow in the presence of electrostatic fields $[6]$.

An extention of this problem is the second approach due to the authors [7, 8], which is based on a Biharmonic equation in two-dimensional (2D) isotropic defect-free media. By discretizating the Biharmonic equation we have proved that a transition from dense to multibranched growth can also be a consequence of a different coupling of displacements during the pattern formation. Within the Biharmonic model the transition appears when the growth velocity at the surfaces present a minimum as also occurs within the Poisson growth. The later implies that both approaches, i.e. Poisson and Biharmonic growth, describe a similar class of complex structural transition phenonema from two different perspectives and on different systems.

It is the aim of this work to deal with a macroscopic characterization of fractals 
showing up a structural transition on growing by using the optical diffraction method. The formalism adopted is not new, as it is an extention of what has been largely applied to analyse optical diffraction from rather 'regular' sctructures like diffusion limited aggregates (DLA) [9] and Laplacian fractals (see, e.g., [10, 11, 12, 13]), besides Koch clusters [14, 15].

We shall expand on these ideas to study the optical diffraction patterns of fractals displaying a transition from dense to multibranched structure. Fractal growth is here numerically simulated using the Poisson and Biharmonic approaches and the results are compared with the well-known cases of DLA and Laplacian growth. We investigate the effect of the structural transition on the diffracted intensity and, consequently, on the fractal dimension $d_{f}$ for all different types of clusters.

For completeness we shall give a brief description of the optical diffraction formalism. Let us start considering a fractal structure composed of $N$ identical and similarly oriented particles on the plane $x-y$. The position of their centers of mass is given by $\mathbf{R}_{n}=$ $\left(x_{n}, y_{n}\right)$, where $n=1, \ldots, N$. In this system we investigate the Fraunhofer diffraction pattern for the fractal, assuming that each particle corresponds to one aperture. This assumption is experimentally realizable even in the case of dark clusters, since they can be photographed on high contrast films which are later lightened [12]. Supposing that the incident plane wave propagates parallel to the $z$-axis, the diffraction amplitude due to $N$ 'apertures' can be expressed as [16]

$$
A(\mathbf{k})=C \int_{S} e^{-i \mathbf{k r}} d^{2} \mathbf{r} \sum_{n=1}^{N} e^{-i \mathbf{k} \mathbf{R}_{n}}
$$

where $C$ is a constant factor, $S$ denotes the surface occupied by one particle. The vector $\mathbf{k}$ in Eq.(1) is the component, parallel to the $x y$-plane, of the scattered wave vector. Its 
modulus is given by $k=\frac{2 \pi}{\lambda} \sin \theta \approx \frac{2 \pi}{\lambda} \theta$, where $\theta$ is the angle (small in practice) which the scattered wave vector makes with the $z$-axis and $\lambda$ is the wave length of the incident light.

The form factor $F(\mathbf{k})$, corresponding to the intensity scattered by one 'aperture', is determined by the integral in Eq.(11). The sum over $n$ in Eq.(11) determines the structure factor

$$
S(\mathbf{k}) \equiv\left|\frac{1}{N} \sum_{n=1}^{N} e^{-i \mathbf{k} \mathbf{R}_{n}}\right|^{2} .
$$

In the case that $k$ is smaller than $a^{-1}$ ( $a$ being the size of an elementary particle), the form factor is practically constant, (i.e., $F(\mathbf{k}) \approx 1$ ) and the light distribution in the diffraction pattern is given by the structure factor such that the normalized diffraction intensity $I(\mathbf{k}) \approx S(\mathbf{k})$, with $k a<1$.

The factor $\mathrm{S}(\mathbf{k})$ of Eq.(2) depends on the distribution of particles in the cluster and is related to its fractal dimension. This relation can be found by using densitydensity correlation functions (see, e.g., [9, 13] and references therein). Accordingly, for $L^{-1}<k<a^{-1}$ ( $L$ is the size of the whole aggregate) the expected value of the intensity -or, alternatively, of the structure factor $S(\mathbf{k})$ - is

$$
<I(\mathbf{k})>=\int d^{2} \mathbf{R} e^{-i \mathbf{k} \mathbf{R}}<\rho\left(\mathbf{R}_{0}\right) \rho\left(\mathbf{R}+\mathbf{R}_{0}\right)>
$$

where

$$
<\rho\left(\mathbf{R}_{0}\right) \rho\left(\mathbf{R}+\mathbf{R}_{0}\right)>=\int d^{2} \mathbf{R}_{0} \rho\left(\mathbf{R}_{0}\right) \rho\left(\mathbf{R}+\mathbf{R}_{0}\right) \sim R^{-\alpha}
$$

represents the density-density correlation function. For fractal aggregates this relation obeys a power law variation [9], where the exponent $\alpha$ is related to the Hausdorff dimension 17$] d_{f}=d-\alpha$ with $d$ the Euclidean space dimension. This variation of the 
density-density correlation function leads to the power law behavior of the diffracted intensity as a function of the wave vector $I(\mathbf{k}) \sim k^{-d_{f}}$ [9]. Thus, it is possible to estimate the fractal dimension $d_{f}$ from diffraction patterns. In particular we shall see that this is valid for fractal structures displaying a structural transition as those at hand.

In the following we shall calculate the quantities $S(\mathbf{k})$ and $\langle I(\mathbf{k})>$ of the diffraction intensity employing Eqs.(2) and (3) and shall search for a power law behaviour. For simplicity we shall assume that the apertures (or particles) have a squared form $a \times a$ with a distribution obtained by numerically simulating fractal growth. In the simulations the particles are distributed on a squared lattice, and the centre-of-mass coordinates $x_{n}$, $y_{n}$ are assumed to take the following discrete values

$$
x_{n}=m_{n} a, m_{n}=0, \pm 1, \pm 2, \ldots ; \quad y_{n}=\ell_{n} a, \quad \ell_{n}=0, \pm 1, \pm 2, \ldots
$$

Therefore, according to Eqs.(2) and (5), the structure factor is calculated by the formula

$$
S\left(k_{x}, k_{y}\right) \equiv\left|\frac{1}{N} \sum_{n=1}^{N} e^{-i a\left(k_{x} m_{n}+k_{y} \ell_{n}\right)}\right|^{2} .
$$

Following the DLA simulation model [9], we add particles to the growing clusters one at the time undergoing a random walk that starts from a point on a variable circle centered on the simulation box. In this process the particles are deposited adjacent to occupied lattice sites to then start off again the random walk of a new particle at another randomly chosen position, and so on. An example of DLA is shown in Fig.1 (upper left-corner).

Laplacian fractals are generated using the growth law (the so-called dielectric breakdown model) [6]

$$
\nabla^{2} \phi=0
$$


We use lattice sites enclosed within a circle of (normalized) radius $r=\sqrt{i^{2}+j^{2}}=100$ such that $\phi^{o}$ and $\phi^{i}$ are unity and zero at the outer circular boundary and the inner growing aggregate, respectively. Seed particles are placed centered in the simulation box. The discretazion procedure then follows standard techniques [6] till solutions of the discretization of Eq.(17) converge to a desidered accuracity. The stochastic growth probability $P$ adopted (at the grid site $(i, j)$ ) is assumed to be proportional to the local field [6], i.e.

$$
P_{i j}=\frac{\left|\phi_{i, j}\right|^{\eta}}{\sum\left|\phi_{i j}\right|^{\eta}}
$$

where the sum runs over nearest neighbor sites to a cluster. Herein we set $\eta=1$ for the sake of simplicity. An example of Laplacian fractal is shown in Fig.1 (upper right-corner).

The effect of screening on fractal structures growing under electrostatic fields was reported in Ref. [3, 田. Within this model the previuos Laplacian equation is replaced by the linearized Poisson-Boltzmann equation

$$
\nabla^{2} \phi=\lambda^{2} \phi
$$

The origin of screening lies in the presence of free charges and leads to a rich variety of patterns -see the example in Fig.1 (lower left-corner). The model introduces a new length scale, i.e., $\lambda$, and a nontrivial dependence on the boundary conditions which is responsible for a structural transition on growing. The patterns can have a fractal character at scales shorter than $\lambda$, be Eden-like, or grow dense, to then follow the transition from dense to single branch growth, which is characterized by a change in the sign of the electrostatic field at the aggregate [3]. However, one of us [5] has demostrated that the transition found using Eq.(9) is altered by the existence of a critical field in the growth of the pattern. 
On the other hand, the alternative Biharmonic model under consideration is based on the discretization (in a square lattice of size $L \times L$ ) of the Biharmonic equation $\square, 8$

$$
\nabla^{2}\left(\nabla^{2} u\right)=0
$$

A crucial difference with respect to the above Laplacian and Poisson models, is that within the Biharmonic model, iterative procedures are carried out around thirteen next nearest neighbours -and not on four as for Eqs.(7) and (96)- on equal grounds. Because of this the formation of connected patterns within the Biharmonic equation becomes non trivial, hence this model is more involved than Laplacian or Poisson growth. To generate Biharmonic fractal patterns -as seen in Fig.1 (lower right-corner)- we set for simplicity the derivative boundary condition, that is necessary along the radial-direction, equal to zero and the growth probability $P$ proportional to $\nabla^{2} u$, (corresponding to the potential in Eq.(8)).

We focus now on the results of diffraction theory. Due to the complexity of the present numerical calculations for growing fractals ( 8 hrs circa of CPU Convex time for each run), our results are based in a statistics of only few different clusters for each class of complex structures considered. The data shown next is displayed for illustrative purposes, whereas the straight lines (used to estimate $d_{f}$ ) are the result of our crude, but representative, statistics.

To this end we can add that the accuracity achievable depends on the wavevector regime considered. Variations of $\langle I(k)\rangle$ decrease on increasing $k$ and, accordingly, the error also decreases. The reason for this essentially lies on the number of particles considered (which form the fractal) and also on the shape of the box used in simulations (squared in the present case). For $k$ values above the transition point $(\sim 1 / 0.6 L)$ we 
estimate the error of our calculations to be less than $1 \%$, whereas for $k$ values near to the system size $\sim 1 / L$ the errors increase to about $5 \%$. Thus, to make a better estimation of $d_{f}$ it will be necessary to obtain statistic for $\langle I(k)>$ over a wide range of $k$-values.

As an example we plot in Fig.2 the diffracted intensity $I$ as a function of the wave vector $\mathbf{k}$ at the plane $k_{y}=0$. The diffraction pattern corresponds to a Biharmonic fractal with 1500 particles (each of size $a$ ) similar to the one displayed in Fig.1. This plot shows strong fluctuations associated to the fractal structure of the aggregate. It can be seen that fluctuations between zeros of the form factor $\left(2 \pi n \leq\left|k_{x}\right| a \leq 2 \pi(n+1)\right.$; $n=1,2, \ldots)$ are of the same type. That is, the structure fractor turns out to be a periodic function of the wave vector:

$$
S\left(k_{x}+2 \pi m / a\right)=S\left(k_{x}\right) ; m=0, \pm 1, \pm 2, \ldots
$$

This fact is a direct consequence of the assumed distribution of particles on a squared lattice (c.f., Eq.(6)). When the magnitude of the wave vector is sufficiently large, namely $k_{x} a \gg 1$, the intensity decreases considerably due to the form factor $F\left(k_{x}\right) \sim k_{x}^{-2}$.

Information about the fractal dimension $d_{f}$ may be obtained at low values of the wave vector $\left(L^{-1} \ll\left|k_{x}\right| \ll a^{-1}\right)$. Therein $F\left(k_{x}\right) \approx 1$ and $I\left(k_{x}\right) \approx S\left(k_{x}\right)$. It is noteworthy that in the case of deterministic fractals [10] the method, used to obtain the fractal dimension $d_{f}$, consists of averaging the structure factor over each of its frequency bands, which are scale invariant. The so averaged structure factor $\left\langle S\left(k_{x}\right)>\right.$ varies according the power law $k_{x}^{-d_{f}}$. In our example the structure factor for a random Biharmonic fractal, as shown in Fig.3, also decreases at $k_{x} a \ll 1$, but it has no scale-invariant frequency bands. Therefore, we cannot apply the preceeding relation between $S\left(k_{x}\right)$ and $k_{x}$ for random fractals. In order to avoid this problem, we have calculated the averaged 
intensity defined by the expression

$$
<I(k)>\equiv \frac{1}{2 \pi} \oint d \phi I(\mathbf{k}) ; \quad k_{x}=k \cos \phi, \quad k_{y}=k \sin \phi
$$

As we shall see next, this quantity has an evident power law behavior as a function of the modulus $(k)$ of the wave vector.

Our numerical results for $\langle I(k)>$ of diffussion limited and Laplacian aggregates are given in Fig. 4 by full and open squares, respectively. The averaged intensity $\langle I(k)>$ is smoother than $I(\mathbf{k})$ and decreases as $k^{-d_{f}}$. The fractal dimension $d_{f}$ is obtained by adjusting the log-log plot of $\langle I(k)>$ to a straight line as indicated in these figures. So, the fractal dimensions for DLA and Laplacian patterns obtained from these curves are $d_{f}=1.72$ and $d_{f}=1.70$, respectively. These $d_{f}$ values are closed to the values calculated via the box counting method, namely, by counting the number of particles $N(r)$ inside an increasing radius $r$ (around a seed particle) -see, e.g., [18].

We shall see next that a structural transition during fractal growth leads to a change in their fractal dimension $d_{f}$. The slopes of the curves in Figs. 5 and 6 show the decrease of the fractal dimension for Poisson and Biharmonic agregates, respectively. (These were roughly estimated similarly to Figs.3 and 4).

In the case of Poisson fractals (as the one in Fig.1: lower left-corner), $d_{f}$ varies from 1.60 -full squares in Fig.5- (by counting $\mathrm{N}=650$ particles before the transition) to 1.44 as deduced by fitting the open squares to a second line. Before the structural transition the $d_{f}$ value for a typical Biharmonic fractal (as shown in Fig.1: lower right-corner) is equal to 1.66 -full squares in Fig.6- being quite closed to the corresponding fractal dimension of diffussion limited agregates and Laplacian fractals in Fig.4, respectively. After the transition point the $d_{f}$ obtained from the slope in Fig.6 (open squares) for $\langle I(k)>$ of 
a Biharmonic pattern reduces to a value of 1.59 .

For circular geometry, a very crude analytical analysis of the Biharmonic Eq.(10) implies that the transition point for Biharmonic fractals is approximately located at a distance far from the center equal to $r_{\ell} /(L / 2) \approx e^{-1 / 2}$ by considering displacements of the growing surfaces in a continuous limit in reasonable agreement with numerical simulations [7, 8]. By comparing the structures in Fig.1, it can be seen that Poisson fractals display a transition point which appears at a rather shorter distance than irreversible Biharmonic structures (the latter enclosed by a circle). This structural transition appears at a point in which the growth velocity of the active zone of the clusters exhibits a minimum similarly to what occurs within Poisson growth, but with different magnitudes [3, 19]. This fact explains the small differences observed in the value of $d_{f}$ after the structural transition within the Poisson and Biharmonic approaches.

Results of optical diffraction thus enables to identify and relate changes in the fractal dimension $d_{f}$ of aggregates to variations in the diffracted intensity as a function of the wave vector. We have seen that there is indeed a decrease of $d_{f}$ above the structural transition, which appears beyond one half of the system size (assuming $\eta=1$ in Eq.(8)) in accord with early crude estimations [7, 8, 19] made using the box counting method, even if it falls beyond the transition point. Different magnitudes of $d_{f}$ above the structural transitions are due to the different growth velocities obtained from the Poisson and Biharmonic approaches.

Our present findings may -in principle- be experimentally confirmed. The averaged intensity $<I(k)>$ might be determined with an experimental arrangement as in the one described in [10]. Therein, a photomultiplier is connected to a multichannel analyzer to thus record $I\left(k_{x}, k_{y}\right)$ and the displacement of the photomultiplier is controlled by a 
high-precision motorized micrometer. Then, after scanning the diffraction patterns, the average of intensity over concentric circles $(<I(k)>)$ is obtained.

Concerning our simulations, it would be interesting to extend them to values of $\eta \neq 1$. This is so because by tuning $\eta \rightarrow 0$, the structural transition now corresponds to a "dense-to-multibranched transition", whereas for $\eta \rightarrow \infty$ one obtains a "transition from slow to faster growth" 19 .

\section{Acknowledgments}

One of the authors, F.P-R., would like to thank the partial support by the Consejo Nacional de Ciencia y Tecnología (CONACyT, México) under Grant No.2048-E-9302. The others two, W.W. and E.C. acknowledge the Scientific Computer Section at ICTPTriste, Italy, for assistance. 


\section{References}

[1] E. Ben-Jacob, H. Shmueli, O. Shochet and A. Tenenbaum, Physica A 187, 378 (1992).

[2] V. Fleury, J.-N. Chazalviel and M. Rosso, Phys. Rev. Lett. 68, 2942 (1992).

[3] E. Louis, F. Guinea, O. Pla and L.M. Sander, Phys. Rev. Lett. 68, 209 (1992).

[4] J. Castella, E. Louis, F. Guinea, O. Pla and L.M. Sander, Phys. Rev. E 47, 2729 (1993).

[5] W. Wang, Phys. Rev. E 47, 2893 (1993).

[6] L. Niemeyer, L. Pietronero and H.J. Wiesmann, Phys. Rev. Lett. 52, 1033 (1984).

[7] W. Wang and E. Canessa, Phys. Rev. E 47, 1243 (1993).

[8] E. Canessa and W. Wang, Z. Naturforsch. 48a, 945 (1993).

[9] T.A. Witten and L.M. Sander, Phys. Rev. B 27, 5686 (1983).

[10] C. Allain and M. Cloitre, Phys. Rev. B 33, 3566 (1986).

[11] B.H. Kaye, in 'A random walk through fractal dimensions, (V.C.H. Verlag, Berlin, 1989).

[12] D. Berger, S. Chamaly, M. Perreau, D. Mercier, P. Monceau and J.C.S. Levy, J. Phys. I (France) 1, 1433 (1991).

[13] G. Korvin, in 'Fractal models in the earth sciences', (Elsevier, Amsterdam, 1992). 
[14] K.E. Peiponen, R. Silvennoinen, J. Uozumi, T. Asakura, M. Savolainen, Optik 93, 91 (1993).

[15] J. Uozumi, H. Kimura, T. Asakura, J. Mod. Opt. 37, 1011 (1990); ibid, 38, 1335 (1991).

[16] M. Born and E. Wolf, in 'Principles of optics', (Pergamon Press, 1975).

[17] B. Mandelbrot, in 'Fractals, form, chance, and dimension', (Freeman, San Francisco, 1977).

[18] E. Canessa and B. Tanatar, Phys. Rev. A 44, 347 (1991).

[19] W. Wang and E. Canessa, submitted for publication. 


\section{Figure captions}

- Fig.1: The different types of fractal aggregates investigated using optical diffraction theory. Diffusion limited agregate with 2000 particles. Laplacian fractal composed of 2500 particles. Poisson fractal (1300 particles) with structural transition. Biharmonic fractal (3000 particles) with structural transition. In the later the circle locates the transition point at $60 \%$ far from the center.

- Fig.2: Central profile $\left(k_{y}=0\right)$ of the diffraction spectrum for a Biharmonic fractal with 1500 particles.

- Fig.3: Structure factor of a typical Biharmonic fractal as in Fig.2.

- Fig.4: Log-log plot of the angle-averaged intensity $<I(k)>$ against $k$ for diffusion limited (full squares) and Laplacian (open squares) agregates with 2000 and 2500 particles, respectively.

- Fig.5: Log-log plot of the angle-averaged $<I(k)>$ against $k$ for a Poisson agregate as in Fig.1. Full squares: before the structural transition (650 particles). Open squares: after the structural transition.

- Fig.6: Log-log plot of the angle-averaged $<I(k)>$ against $k$ for a Biharmonic fractal as in Fig.1. Full squares: before the structural transition (1500 particles). Open squares: after the structural transition. 